\title{
Complicaciones cerebrovasculares luego de angiografía coronaria en contexto de infarto agudo de miocardio. Estudio de cohorte
}

\author{
Dres. Gimena Loza, Valeria Rocha, Juan Albistur, María Florencia Brunet, \\ Heber Hackembruch, Andrés Gaye
}

\section{Resumen}

Introducción: los eventos cerebrovasculares son una complicación grave, pero infrecuente, de los procedimientos coronarios invasivos. La angioplastia coronaria aumenta el riesgo de presentarla en 17 veces. Los pacientes que la sufren tienen más complicaciones agregadas y mayores tasas de mortalidad.

Objetivo: determinar las características clínicas y evolutivas de los pacientes que desarrollaron un evento cerebrovascular luego de una angiografía coronaria de urgencia en el contexto de un infarto agudo de miocardio (IAM). Secundariamente, analizar la relación temporal entre ambos eventos e identificar factores previos asociados al desarrollo de la complicación neurológica.

Método: cohorte retrospectiva de pacientes que sufrieron un evento cerebrovascular de cualquier tipo dentro de los 30 días posteriores a un procedimiento coronario por IAM en el Centro Cardiovascular Universitario, entre 2008 y 2017. Se analizaron características demográficas, del procedimiento coronario y del evento cerebrovascular.

Resultados: 24 pacientes, 54,2\% hombres; 1/3 del total tenía ataque cerebrovascular (ACV) previo. El 66,7\% sufrió IAM sin elevación del segmento ST. Todos los eventos cerebrovasculares fueron isquémicos; la mayoría sobre la circulación anterior. El 62\% ocurrió en las primeras 48 horas luego del procedimiento coronario, asociándose a mayor duración del procedimiento y número de vasos tratados. En tres casos se utilizó activador tisular del plasminógeno recombinante para reperfusión del evento neurológico. La mediana de NIHSS (National Institute of Health Stroke Scale) fue de 4 puntos (IQ 2-8) en IAM sin elevación del ST, y 8 puntos (IQ 3-20) en IAM con elevación del ST ( $\mathrm{p}=0,20)$. El 79\% de los pacientes sufrió complicaciones adicionales durante la hospitalización; cinco fallecieron.

Conclusiones: entre quienes sufrieron complicaciones cerebrovasculares luego de angiografía coronaria pos-IAM existió una proporción similar de ambos sexos y un porcentaje elevado de pacientes con antecedentes de ACV. La forma de presentación del evento cardiovascular fue predominantemente IAM sin elevación del ST. Los eventos cerebrovasculares fueron isquémicos, en su mayoría de la circulación cerebral anterior y ocurrieron en las primeras 48 horas. El ACV/AIT (ataque isquémico transitorio) en este contexto podría asociarse a una mayor estancia hospitalaria y mortalidad.

Palabras clave: $\quad$ ANGIOGRAFÍA CORONARIA

ACCIDENTE CEREBROVASCULAR

ATAQUE ISQUÉMICO TRANSITORIO

INFARTO DEL MIOCARDIO

ESTUDIOS DE COHORTES

\section{Cerebrovascular complications after invasive percutaneous coronary procedure for acute myocardial infarction in a patient cohort}

\section{Summary}

Introduction: cerebrovascular events are serious but infrequent complications of invasive percutaneous coronary procedure. Coronary angioplasty increases the risk 17 times. Patients suffering this complication have higher morbidity and mortality rates.

Objective: to determine the clinical and evolutionary characteristics of the patients who developed a cerebrovascular event after an emergency coronary angiography in the context of an acute myocardial infarction. Secondly, analyze the

Centro Cardiovascular Universitario, Hospital de Clínicas. Montevideo, Uruguay.

Correspondencia: Dra. Gimena Loza Rossi. Correo electrónico: gime.loza@gmail.com

Los autores declaran no tener conflictos de intereses. Este trabajo no tiene fuentes de financiamiento. 
temporal relationship between both events and identify previous factors associated with the development of neurological complications.

Methods: demographic, characteristics of the angiographic procedure, and cerebrovascular features of a population of patients with stroke occurring within 30 days after of invasive percutaneous coronary procedure for myocardial infarctions are described. Data was retrospectively collected from the Cardiovascular University Center (Hospital de Clínicas, Montevideo, Uruguay) between 2008-2017.

Results: 24 patients, $54.2 \%$ were men; $1 / 3$ had prior stroke; $66.7 \%$ presented non ST segment elevation myocardial infarction. All of the cerebrovascular events were ischemic, most were from the anterior circulation. $62 \%$ occurred in the first 48 hours after invasive percutaneous coronary procedure, having this group a higher percentage of percutaneous coronary angioplasty and longer procedures. In three patients recombinant tissue plasminogen activator was used for stroke treatment. NIHSS median was 4 points (IQ 2-8) in patients without ST-segment elevation myocardial infarction and 8 points (IQ 3-20) in patients with ST-segment elevation myocardial infarction $(p=0.20)$. 79\% of patients had complications during the hospitalization, and 5 died.

Conclusions: there was a similar proportion of men and women, and a high percentage of patients with a history of stroke. The presentation of the cardiovascular events was predominantly non ST myocardial infarction. All the cerebrovascular events were ischemic, it occurred for most patients in the first 48 hours after invasive percutaneous coronary procedure, and the anterior brain circulation was most commonly affected. Stroke/transient ischemic attack after invasive percutaneous coronary procedure could be associated with longer hospital stays and death.

Key words:

CORONARY ANGIOGRAPHY
STROKE
TRANSIENT ISCHEMIC ATTACK
MYOCARDIAL INFARCTION
COHORT STUDIES

\section{Complicações cerebro-vasculares após procedimento coronário invasivo percutâneo para infarto do miocárdio aguda em um coorte do paciente}

\section{Resumo}

Introdução: o acidente vascular cerebral é uma complicação séria, mas pouco frequente, da procedimiento coronário invasivo percutâneo. Angioplastia coronariana aumenta o risco 17 vezes. Pacientes que sofrem dessa complicação têm mais complicações e maiores taxas de mortalidade.

Objetivo: determinar as características clínicas e evolutivas dos pacientes que desenvolveram um evento cerebrovascular após uma angiografia coronária de emergência no contexto de um infarto agudo do miocárdio. Em segundo lugar, analise a relação temporal entre os dois eventos e identifique os fatores anteriores associados ao desenvolvimento de complicações neurológicas.

Métodos: uma coorte retrospectiva dos pacientes que sofreram um acidente vascular cerebral nos primeiros 30 dias de um procedimento coronário para o infarto agudo do miocárdio, e a análise dos seus dados demográficos e características de processo coronária e acidente vascular cerebral é realizada é descrito. Dados do Centro Cardiovascular Universitário do Hospital de Clínicas de Montevidéu, Uruguai, foram obtidos retrospectivamente a partir das datas entre 2008 e 2017.

Resultados: 24 pacientes; $54,2 \%$ dos pacientes eram homens; $1 / 3$ haviam sofrido um ataque cerebrovascular prévio; $66,7 \%$ apresentavam infarto agudo do miocárdio sem supradesnivelamento do segmento ST. Todos os eventos cerebrovasculares foram isquêmicos, a maioria era da circulação anterior. $62 \%$ dos eventos cerebrovasculares ocorreram nas primeiras 48 horas após o procedimento coronário, tendo encontrado nesse grupo procedimentos mais longos e com mais angioplastias coronarianas realizadas. Em 3 pacientes, a ativador do plasminogênio tissular recombinante foi usada para tratar o acidente vascular cerebral. O NIHSS mediana foi de 4 pontos (IQ 2-8) no infarto agudo do miocárdio, sem elevação do segmento ST, e 8 pontos (IQ 3- 20) no infarto agudo do miocárdio com elevação do segmento ST $(\mathrm{p}=0,20)$. 79\% dos pacientes sofreram complicações durante a internação e 5 morreram.

Conclusões: houve uma proporção semelhante de ambos sexos e uma alta porcentagem de pacientes com história de acidente vascular cerebral. A apresentação do evento cardiovascular foi predominantemente IAM sem supradesnivelamento do segmento ST. O ataque cerebrovascular foi isquêmico, ocorreu mais freqüentemente nas primeiras 48 horas e afetou principalmente a circulação cerebral anterior. Acidente vascular cerebral após intervenção coronária percutânea poderia estar associada a uma permanência hospitalar mais longa e mortalidade.

Palavras chave: $\quad$ ANGIOGRAFIA CORONÁRIA

ACIDENTE VASCULAR CEREBRAL

ATAQUE ISQUÊMICO TRANSITÓRIO

INFARTO DO MIOCÁRDIO

ESTUDOS DE COORTES 


\section{Introducción}

Desde el inicio de la cardiología intervencionista ${ }^{(1)}$, tanto la cineangiocoronariografía (CACG) como la angioplastia coronaria (ATC) han ido evolucionando hasta convertirse, la primera, en el estándar oro para el diagnóstico, y la segunda, en el método invasivo más frecuentemente utilizado como tratamiento.

En Estados Unidos se realizan más de 2 millones de CACG por año(2), lo que corresponde aproximadamente a un procedimiento cada 157 habitantes por año. En Uruguay, en el año 2017, se realizó un procedimiento cada 427 habitantes ${ }^{(3)}$. Simultáneamente se ha observado un incremento de aproximadamente $55 \%$ en la cantidad de intervenciones coronarias percutáneas (ICP) realizadas desde el año 2004 al 2016. $\mathrm{El}$ aumento del número de ICP determina que las complicaciones asociadas se observen con mayor frecuencia. Estas pueden ir desde complicaciones menores, como el hematoma en el sitio de punción o la bradicardia transitoria frente a la inyección de contraste, hasta complicaciones mayores como la perforación cardíaca, cierre brusco de una arteria coronaria durante la ATC, ataque cerebrovascular (ACV), ataque isquémico transitorio (AIT), insuficiencia renal o muerte $(<1 \%)^{(1)}$.

El ACV y el AIT son complicaciones temibles, con una incidencia reportada entre $0,07 \%$ y $1 \%(4-12)$. La ICP incrementa 17 veces el riesgo de presentarlas dentro de las primeras 48 horas del procedimiento $^{(13)}$.

Se han descrito factores que aumentan el riesgo de ACV/AIT asociado a ICP, vinculado esto a características tanto del paciente como del procedimiento. Entre las primeras se destacan: edad avanzada, sexo femenino, diabetes mellitus (DM), hipertensión arterial (HTA), enfermedad renal crónica (ERC) o enfermedad cerebrovascular previa, y fracción de eyección del ventrículo izquierdo (FEVI) disminuida. En cuanto a las características del procedimiento, se asocian con mayor riesgo los procedimientos más largos, con mayor volumen de contraste, utilización de catéteres más rígidos y de mayor calibre, procedimientos de emergencia, trombectomía, ATC sobre puentes venosos o sobre lesiones más complejas y uso de balón de contrapulsación intraaórtico (BCIA)(2,4-7,10,11,14-18).

La importancia de esta complicación radica en la morbimortalidad que conlleva. Se evidencia un aumento de la mortalidad intrahospitalaria y a un año desde 1\% en pacientes sin ACV hasta 20\%-30\% en los que sí lo presentan, aumentando además las complicaciones intrahospitalarias, como insuficiencia renal aguda, necesidad de diálisis y mayor índice de discapacidad ${ }^{(4-6,10)}$.

\section{Principales aportes}

Los eventos cerebrovasculares son una complicación infrecuente de los procedimientos coronarios percutáneos, y se asocian a otras complicaciones y mortalidad. Se analiza la relación entre ambos eventos en una población de pacientes pos-IAM, sus características demográficas y los factores de riesgo para la complicación neurológica.

En nuestro país no existen trabajos que establezcan las características de los pacientes que sufren un ACV o AIT durante los 30 días siguientes a una ICP, así como tampoco datos sobre el seguimiento de dichos casos.

El objetivo de este trabajo es determinar las características clínicas, forma de presentación (tanto del evento neurológico como del evento cardíaco) y evolución intrahospitalaria de los pacientes que desarrollan un evento cerebrovascular luego de una CACG de urgencia con o sin ICP terapéutica en el contexto de un IAM. Como objetivo secundario se plantea analizar la relación temporal entre ambos eventos e identificar factores asociados al desarrollo de ACV/AIT en las primeras 48 horas posteriores al procedimiento coronario.

\section{Método}

Se trata de un estudio observacional, descriptivo-analítico, retrospectivo, que evalúa la ocurrencia de un evento cerebrovascular agudo como complicación de una CACG con o sin ATC realizada en el contexto de un evento coronario agudo, en el período comprendido entre enero de 2008 y diciembre de 2017 en pacientes asistidos en el Centro Cardiovascular Universitario (CCVU) del Hospital de Clínicas.

Se incluyeron pacientes mayores de 18 años que desarrollaron un ACV o un AIT en un período menor a 30 días desde el procedimiento ${ }^{(13)}$.

Definimos ACV isquémico como la presencia de un episodio de disfunción neurológica resultante de isquemia focal cerebral o espinal persistente, asociada a evidencia imagenológica de infarto. Definimos ACV hemorrágico como la presencia de un episodio de disfunción neurológica resultante de hemorragia focal cerebral o espinal asociada a evidencia imagenológica de sangrado. A su vez, definimos AIT como la disfunción neurológica o retiniana resultante de isquemia focal transitoria, de menos de una hora de duración, y que no se acompaña de evidencia de infarto cerebral en la imagenología $(4,5,19,20)$. 
Tabla 1. Características generales de la población. $\mathrm{n}=24$

\begin{tabular}{lc}
\hline Edad (años)** & $65,5(59,0-75,7)$ \\
Sexo femenino* & $11(45,8 \%)$ \\
Raza blanca* & $21(87,5 \%)$ \\
\hline Hipertensión arterial* & $19(79,2 \%)$ \\
\hline Fibrilación auricular* & $2(8,3 \%)$ \\
\hline Diabetes mellitus* & $4(16,7 \%)$ \\
Tabaquismo actual o pasado* & $17(70,8 \%)$ \\
\hline Dislipemia* & $11(45,8 \%)$ \\
Alcoholismo* & $4(16,7 \%)$ \\
Ángor estable previo* & $7(29,2 \%)$ \\
\hline Infarto de miocardio previo* & $6(25,0 \%)$ \\
Ataque cerebrovascular previo** & $9(37,5 \%)$ \\
\hline Enfermedad renal crónica* & $1(4,2 \%)$ \\
FEVI \%** & $46(35,0-55,0)$ \\
\hline *n (\%); ** mediana (IQ). FEVI: fracción de eyección del ven- \\
trículo izquierdo
\end{tabular}

Se definió ACV/AIT posterior al procedimiento coronario como aquel que se produjo dentro de los 30 días del evento ${ }^{(6)}$. Se denominó ACV/AIT precoz o tardío según se produjera en las primeras 48 horas o posteriormente ${ }^{(13)}$. Se definió IAM según la cuarta definición universal y se clasificó con y sin elevación del segmento ST de acuerdo a las guías actuales ${ }^{(21)}$

Se excluyeron los casos en que se desarrolló el ACV/AIT en primer lugar y luego el evento coronario, así como aquellos pacientes en que no fue posible establecer el orden temporal entre ambos eventos, o éstos se dieron en forma simultánea. Además, se excluyeron los que presentaron una complicación cerebrovascular como consecuencia de un procedimiento coronario realizado de coordinación, luego de procedimientos cardiológicos intervencionistas no coronarios y en los casos en que el evento neurológico se dio en el posoperatorio de cirugía cardíaca.

Se realizó una búsqueda en la base de datos del Servicio de Hemodinamia del CCVU y en las historias de ingreso y egreso, utilizando las palabras clave "ACV, AIT, neurológico, stroke" para la búsqueda de casos. Se revisaron los protocolos de la unidad de ACV del período y se seleccionaron aquellos en los cuales existían datos de un evento coronario en los 30 días previos al evento cerebrovascular. Con dicha información se elaboró una lista de casos índice para posteriormente revisar exhaustivamente las historias clínicas y determinar si cumplían los criterios de inclusión al estudio. Con los que cum- plían los criterios de inclusión se elaboró una base de datos con variables clínicas, paraclínicas y de seguimiento. Todas las variables de estudio fueron recogidas por los autores, retrospectivamente, desde las historias clínicas. El estudio fue aprobado por el Comité de Ética de la Investigación del Hospital de Clínicas.

\section{Análisis estadístico}

Se describieron las variables cuantitativas continuas utilizando mediana e intervalo intercuartilo (IQ). Las variables cualitativas se expresaron en valor absoluto y porcentaje. Para la escala de NIHSS al ingreso y egreso de los pacientes con ACV/AIT y en la comparación entre IAM con elevación del segmento ST (IAMCEST) e IAM sin elevación del segmento ST (IAMSEST) se utilizó test de Wilcoxon para variables dependientes. El nivel de significación es en todos los casos $\alpha<0,05$.

Se utilizó para el análisis el paquete estadístico IBM SPSS Statistics versión 22.

\section{Resultados}

Se analizaron 83 historias clínicas en cuyo registro figura la presencia de AIT/ACV e IAM con menos de 30 días de diferencia. Del total, 24 pacientes cumplieron los criterios de inclusión. De ellos, 13 eran hombres (54,2\%). La mediana de edad fue 65,5 años (IQ 59-76). Las características demográficas se describen en la tabla 1.

\section{Evento cardiovascular y procedimiento coronario}

El motivo de la CACG fue IAMSEST en 16 casos e IAMCEST en 8 casos. En 17 pacientes $(70,8 \%)$ se realizó algún procedimiento terapéutico (tabla 2). En dos de esos casos solo se realizó ATC con balón (un caso por ATC fallida y otro por deterioro de conciencia que obligó a suspender el procedimiento). Ambas situaciones se dieron en procedimientos terapéuticos sobre puentes venosos. Entre los pacientes que no recibieron tratamiento percutáneo, cinco tenían anatomía coronaria compleja, por lo que se derivaron a cirugía cardíaca, y dos no tenían estenosis coronarias angiográficamente significativas.

En un paciente se realizó implante de stent en la arteria subclavia, como consecuencia de disección iatrogénica de esta durante el procedimiento. No se utilizó BCIA en ningún caso. Se realizó trombectomía manual en cinco casos con IAMCEST y en dos con IAMSEST. Otras características asociadas al procedimiento se describen en la tabla 2. 


\section{Evento cerebrovascular}

Dieciocho pacientes (75\%) sufrieron un ACV isquémico, mientras que 6 pacientes $(25 \%)$ sufrieron un AIT. No hubo ningún ACV hemorrágico.

En cuanto a la severidad inicial del evento cerebrovascular medido por escala de NIHSS, la mediana fue de 3 (IQ 2-8,5, datos disponibles en 17 de 24 casos). Cuando se analiza la severidad de la complicación cerebrovascular según el evento coronario, los ACV/AIT que ocurrieron luego de un IAMCEST presentaron una mediana de la escala de NIHSS de 8 puntos (IQ 3-20), mientras que en los pacientes que presentaron un ACV/AIT luego de un IAMSEST fue de 4 puntos (IQ 2-8), sin diferencia estadísticamente significativa $(p=0,315)$. Se observó una diferencia significativa entre el NIHSS medido al diagnóstico del cuadro neurológico y el NIHSS al alta (3 IQ 2-8,5 vs 0 IQ 0-1,25, p=0,007).

En cuanto al estudio de la etiología cardioaortoembólica del ACV/AIT durante la internación, se observó un caso de trombo en el ventrículo izquierdo, un caso de trombo en orejuela izquierda y un caso de ateromatosis en aorta ascendente. No se observaron casos de estenosis valvular aórtica significativa. En el Doppler de vasos de cuello realizado como parte del estudio etiológico, se encontró ateromatosis en 15/18 casos (dos de ellos con estenosis mayor a 50\%) y un caso sin ateromatosis. En seis pacientes no se contaba con este dato.

Si se considera la clasificación TOAST, $65 \%$ de los pacientes fueron clasificados como de etiología cardioembólica, $4 \%$ aterosclerosis de grandes arterias, $22 \%$ otra causa, y $9 \%$ de causa indeterminada por estudios incompletos.

Tanto en los casos de IAMCEST como en los casos de IAMSEST se observó una mayor incidencia de ACV isquémico versus AIT (87,5\% para IAMCEST y $69 \%$ para IAMSEST).

En cuanto a la relación temporal entre el procedimiento coronario y el ACV/AIT, la mediana en horas fue de 48 (IQ 24-72), con un intervalo entre 0 y 336 horas; 15 pacientes $(62,5 \%)$ tuvieron ACV/AIT en las primeras 48 horas del procedimiento (ACV/ AIT precoz) y $9(37,5 \%)$ luego de las 48 horas hasta 30 días (ACV/AIT tardío).

Dentro del grupo de ACV/AIT precoz, en 12/15 se realizó un procedimiento terapéutico (dos ATC con balón, seis con stent y cuatro con balón y stent); 2/15 pacientes tenían anatomía coronaria con indicación de cirugía de revascularización miocárdica (CRM), y 1/15 no tenía estenosis angiográficamente significativa. Las dos ATC sobre puentes venosos se registraron en este grupo de pacientes.

\begin{tabular}{|c|c|c|}
\hline \multirow{2}{*}{$\begin{array}{l}\text { Forma de } \\
\text { presentación }\end{array}$} & IAMSEST* & $16(66,7 \%)$ \\
\hline & IAMCEST* & $8(33,3 \%)$ \\
\hline \multirow{2}{*}{$\begin{array}{l}\text { Killip y Kimball del } \\
\text { evento coronario** }\end{array}$} & I & $20(83,3 \%)$ \\
\hline & II & $4(16,7 \%)$ \\
\hline \multicolumn{2}{|c|}{ Fibrinolíticos para el evento coronario* } & $2(8,3 \%)$ \\
\hline \multirow{3}{*}{$\begin{array}{l}\text { Angioplastia } \\
\text { coronaria con balón } \\
\text { o stent* }\end{array}$} & Puente venoso & $2(8,3 \%)$ \\
\hline & Un vaso nativo & $12(50,0 \%)$ \\
\hline & Dos vasos nativos & $3(12,5 \%)$ \\
\hline \multicolumn{2}{|c|}{ Número de stents utilizados* } & $2(1-2)$ \\
\hline \multicolumn{2}{|c|}{ Cantidad de contraste $(\mathrm{ml}) * *$} & $210,0(172,5-250,0)$ \\
\hline \multicolumn{2}{|c|}{ Tiempo de radioscopía (minutos) $* *$} & $11,0(9,1-15,6)$ \\
\hline \multicolumn{2}{|c|}{ Trombectomía manual* } & $7(29,2 \%)$ \\
\hline \multirow[t]{3}{*}{ Acceso arterial* } & Radial & $19(79,2 \%)$ \\
\hline & Femoral & $3(12,5 \%)$ \\
\hline & Sin datos & $2(8,3 \%)$ \\
\hline
\end{tabular}

De los nueve pacientes con ACV/AIT tardío, cabe destacar que en cinco se realizó procedimiento terapéutico.

Las características del procedimiento de acuerdo a la relación temporal CACG-ACV/AIT se describen en la tabla 4 .

En 3/24 pacientes se hizo tratamiento trombolítico con rTPA para el evento neurovascular. Todos habían sufrido el ACV isquémico en las primeras 24 horas de la CACG, siendo 110 minutos la mediana de tiempo síntoma-aguja (intervalo 90-115 minutos). Se registró un ACV isquémico leve con NIHSS 5 , uno moderado con NIHSS 10 , y uno grave con NIHSS 32 y coma primario. Hubo un caso con sangrado del acceso arterial radial, el cual cedió con medidas de compresión local. Todos los pacientes que recibieron rTPA tuvieron una buena evolución neurológica con un NIHSS al alta de 0 .

\section{Resultados intrahospitalarios}

La mediana de internación para todos los pacientes fue 8,5 días (IQ 5,2-21,5). Se registraron complicaciones diferentes al ataque cerebrovascular durante la internación en 19 de los 24 pacientes $(79,2 \%)$, dentro de las que se incluyen complicaciones infecciosas, insuficiencia renal con o sin requerimiento de diálisis e insuficiencia cardíaca; $4 / 24$ requirieron internación en unidad de cuidados intensivos. Cua- 
Tabla 3. Datos del evento cerebrovascular. $n=24$

\begin{tabular}{|c|c|c|}
\hline \multirow[t]{3}{*}{$\begin{array}{l}\text { Tipo de evento } \\
\text { neurovascular * }\end{array}$} & $\begin{array}{l}\text { Ataque isquémico } \\
\text { transitorio }\end{array}$ & $6(25 \%)$ \\
\hline & $\begin{array}{l}\text { Ataque cerebrovascular } \\
\text { isquémico }\end{array}$ & $18(75 \%)$ \\
\hline & $\begin{array}{l}\text { Ataque cerebrovascular } \\
\text { hemorrágico }\end{array}$ & $0,0(0 \%)$ \\
\hline \multirow{5}{*}{$\begin{array}{l}\text { Localización } \\
\text { anatómica * }\end{array}$} & Circulación anterior & $19(79,2 \%)$ \\
\hline & TACI & $4(16,8 \%)$ \\
\hline & PACI & $12(50,0 \%)$ \\
\hline & LACI & $3(12,5 \%)$ \\
\hline & Circulación posterior & $5(20,8 \%)$ \\
\hline NIHSS ** & & $3(2,0-8,5)$ \\
\hline
\end{tabular}

tro fallecieron durante la internación y uno de ellos a los tres meses debido a una hemorragia intracraneana bajo tratamiento con anticoagulantes.

\section{Discusión}

Este es el primer estudio publicado a nivel nacional que aborda el ACV/AIT posterior a una ICP en el contexto de un IAM.

En la literatura se describen factores de riesgo para complicaciones cerebrovasculares pos-ICP que podemos dividir en asociados al paciente y asociados al procedimiento.

En cuanto a los primeros, encontramos una alta prevalencia de HTA (>80\%) y aproximadamente un tercio de los casos presentaban historia de ACV previo. El sexo femenino es otro factor de riesgo, lo cual podría explicar, al menos en parte, la mayor proporción de mujeres en nuestra serie $(45,8 \%)$ en comparación con otras series de pacientes con IAM (que oscilan entre $25 \%$ y $30 \%)^{(13,22)}$. Por otra parte, llamativamente se observó una baja prevalencia de DM y ERC (factores de riesgo también asociados con ACV pos-ICP $)^{(5,6,19)}$. Otros factores de riesgo descritos, como la edad mayor a 70 años y la FEVI menor de $30 \%$, no se evidenciaron en nuestro estudio ${ }^{(14)}$.

En cuanto a los factores del procedimiento coronario, solo se incluyeron pacientes que recibieron procedimiento coronario de urgencia, lo cual constituye un factor de riesgo conocido para ACV/AIT. A su vez, procedimientos más complejos, con un mayor tiempo de radioscopía, mayor volumen de contraste y mayor número de stents, han sido vinculados a un mayor riesgo de esta complicación ${ }^{(5,15,19)}$. Sin embargo, en nuestro estudio la mediana del tiempo de radioscopía y el volumen de contraste utilizado son similares a los reportados en pacientes que reciben CACG de urgencia en nuestro centro y no presentaron dicha complicación $^{(23)}$. Otro factor de riesgo es la realización de trombectomía manual ${ }^{(17,24)}$, la cual se observó en un alto porcentaje de casos en nuestra serie $(62,5 \%$ de los casos de IAMCEST).

Dos casos de eventos neurovasculares se produjeron en sujetos que se sometieron a ATC de puente venoso, siendo este otro factor de riesgo conocido para esta complicación ${ }^{(4,5,15,19)}$. La proporción de IAMCEST y IAMSEST observada fue similar a la reportada habitualmente en pacientes que reciben CACG de urgencia.

En cuanto al evento cerebrovascular, se observó que $75 \%$ fueron $\mathrm{ACV}$ isquémicos y $25 \% \mathrm{AIT}$, lo cual se encuentra en concordancia con trabajos previos. No ocurrieron ACV hemorrágicos, en concordancia con la amplia superioridad de frecuencia de las complicaciones isquémicas por sobre los san-

Tabla 4. Características del procedimiento de acuerdo a la relación temporal CACG - ACV/AIT. n=24

\begin{tabular}{|c|c|c|c|}
\hline & ACV precoz. $(n=15)$ & $A C V$ tardio $(n=9)$ & valor $p$ \\
\hline IAMCEST* & $4(26,7 \%)$ & $4(44,4 \%)$ & 0,41 \\
\hline IAMSEST* & $11(73,3 \%)$ & $5(55,6 \%)$ & 0,41 \\
\hline $\begin{array}{l}\text { Tiempo de radioscopía } \\
\text { (minutos)** }\end{array}$ & $13,5(9,0-18,5)$ & $10,0(7,7-11,5)$ & 0,18 \\
\hline Volumen de contraste $(\mathrm{ml})^{* *}$ & $210,0(142,5-250,0)$ & $225,0(185,0-287,5)$ & 0,78 \\
\hline Trombectomía manual * & $3(20,0 \%)$ & $4(44,4 \%)$ & 0,36 \\
\hline $\begin{array}{l}\text { Angioplastia de puente } \\
\text { venoso * }\end{array}$ & $2(13,3 \%)$ & $0 \%$ & 0,51 \\
\hline $\begin{array}{l}\text { Angioplastia durante el } \\
\text { procedimiento * }\end{array}$ & $12(80,0 \%)$ & $5(55,5 \%)$ & 0,36 \\
\hline
\end{tabular}


$\operatorname{grados}^{(5,9,19,25)}$. En cuanto a los mecanismos fisiopatológicos de ACV/AIT posterior a una ICP, se destacan la embolia de material aterotrombótico y la disección vascular ${ }^{(26,27)}$, lo cual explica el bajo porcentaje de infartos lacunares que encontramos en comparación con otras series nacionales ${ }^{(28)}$.

Solo $65 \%$ de los eventos fueron clasificados como de etiología cardioaortoembólica según los criterios TOAST, lo cual supera los porcentajes de cardioembolia en series de ACV fuera del contexto de ICP. Sin embargo, dada la estrecha asociación temporal entre el procedimiento coronario y el evento neurológico, creemos que la mayoría de los casos deberían considerarse de etiología cardioaortoembólica, por lo cual esta clasificación mostraría debilidades en la determinación etiológica de este subgrupo especial de pacientes.

Respecto a la severidad de los eventos, la mayoría se correspondieron a ACV/AIT leves (NIHSS < 10 puntos). A su vez, observamos un buen pronóstico funcional del evento, con una mejoría significativa del puntaje al alta. Esto podría deberse al diagnóstico precoz y a la cooperación entre el equipo de cardiólogos y neurólogos, así como a la predominancia de eventos cerebrovasculares leves. En tres pacientes se administró rTPA como tratamiento de la complicación neurológica, teniendo excelente evolución clínica (NIHSS al alta igual a 0).

El 62,5\% presentó el evento cerebrovascular en las 48 horas posteriores a la ICP. Este grupo de pacientes tuvo mayor porcentaje de procedimientos terapéuticos durante la CACG. El bajo poder estadístico de nuestro trabajo podría explicar la no existencia de diferencias estadísticamente significativas.

La estancia hospitalaria observada fue mayor que la esperada según datos regionales (5,9 vs 8,5 días) ${ }^{(28)}$, seguramente por el hecho de haber presentado un ACV o AIT pos-ICP. La mortalidad hospitalaria fue más alta que la esperada según la escala Killip y Kimball(29) y mayor a la observada en series nacionales de IAM ${ }^{(30)}$. Según el estudio de Camejo y colaboradores $^{(28)}$, la mortalidad de 784 pacientes con ACV/AIT de la unidad de ACV del Hospital de Clínicas fue $11 \%$ a los 30 días, menor a la observada en nuestro estudio. Esto reafirmaría que la conjunción de ambos eventos empobrece el pronóstico cuando lo comparamos con una importante cohorte histórica de pacientes con ACV/AIT en el mismo centro asistencial.

\section{Limitaciones del estudio}

Se trata de un estudio retrospectivo, con las limitaciones inherentes a dicho diseño. En relación con ello, es posible que exista un subregistro de algunas variables, y que no se haya registrado la totalidad de casos de ACV y AIT pos-ICP, dado que existen pa- cientes que retornan a su centro de origen a las 48 horas luego de realizarse un procedimiento coronario invasivo. A su vez, el bajo número de casos registrados determina un menor poder estadístico.

\section{Conclusiones}

Entre quienes sufrieron complicaciones cerebrovasculares luego de angiografía coronaria pos-IAM existió una proporción similar de ambos sexos y un porcentaje elevado de pacientes con antecedentes de ACV. La proporción de IAM con y sin elevación del segmento ST es similar a la reportada internacionalmente.

El evento cerebrovascular fue isquémico en todos los casos y se presentó en su mayoría en las primeras 48 horas posteriores a la ICP. La circulación cerebral anterior fue la predominantemente afectada y la mayoría de los ACV/AIT fueron leves. Algunas características del procedimiento fueron más frecuentes en los eventos cerebrovasculares más precoces (menores a 48 horas), pero estos datos no fueron estadísticamente significativos. Estudios con mayor cantidad de pacientes serían necesarios para evaluar esta asociación. El ACV/AIT consecutivo a una ICP urgente conlleva mayor estancia hospitalaria y mortalidad que el reportado en una serie general de pacientes con ACV/AIT del mismo centro asistencial.

Contribución de autores

Gimena Loza, https://orcid.org/0000-0001-9555-7431

recolección de datos, análisis, discusión de resultados, redacción y respuesta a editores.

Valeria Rocha, https://orcid.org/0000-0001-8380-938X recolección de datos, análisis, discusión de resultados, redacción y respuesta a editores.

Juan Albistur, https://orcid.org/0000-0002-0282-5263 recolección de datos, análisis estadístico, redacción, discusión de resultados y respuesta a editores.

\section{Maria Florencia Brunet,}

https://orcid.org/0000-0002-3591-8028 recolección de datos y discusión de resultados.

Heber Hackembruch,

https://orcid.org/0000-0002-6256-3998 participación en análisis estadístico.

Andrés Gaye, https://orcid.org/0000-0001-7557-3231 discusión de resultados y redacción.

Se declara que las autoras Gimena Loza y Valeria Rocha tuvieron igual participación en el trabajo.

Este artículo fue aceptado para su publicación por: Editor jefe Dr. Gerardo Soca. 


\section{Bibliografía}

1. Moscucci M. Grossman \& Baim's, cardiac catheterization, angiography and intervention. 8 ed. Philadelphia: Lippincott Williams \& Wolters Kluwer, 2014.

2. Werner N, Zahn R, Zeymer U. Stroke in patients undergoing coronary angiography and percutaneous coronary intervention: incidence, predictors, outcome and therapeutic options. Expert Rev Cardiovasc Ther. 2012; 10(10):1297-305. doi: 10.1586/erc.12.78

3. Fondo Nacional de Recursos. Estadísticas médicas 2016. Montevideo FNR, 2016. Disponible en: http:// www.fnr.gub.uy/estadisticas_2016. [Consulta: 10 Set 2019].

4. Fuchs S, Stabile E, Kinnaird T, Mintz G, Gruberg L, Canos D, et al. Stroke complicating percutaneous coronary interventions: incidence, predictors, and prognostic implications. Circulation 2002 106(1):86-91. doi: 10.1161/01.cir.0000020678.16325.e0

5. Dukkipati S, O’Neill W, Harjai K, Sanders W, Deo D, Boura J, et al. Characteristics of cerebrovascular accidents after percutaneous coronary interventions. J Am Coll Cardiol 2004; 43(7):1161-7. doi: 10.1016/j.jacc.2003.11.033

6. Aggarwal A, Dai D, Rumsfeld J, Klein L, Roe M, et al. Incidence and predictors of stroke associated with percutaneous coronary intervention. Am J Cardiol 2009; 104(3):349-53. doi: 10.1016/j.amjcard.2009. 03.046

7. Lazar J, Uretsky B, Denys B, Reddy P, Counihan P, Ragosta M. Predisposing risk factors and natural history of acute neurologic complications of left-sided cardiac catheterization. Am J Cardiol. 1995; 75(15): 1056-60. doi: 10.1016/s0002-9149(99)80724-3

8. Myint P, Kwok C, Roffe C, Kontopantelis E, Za$\operatorname{man} A$, Berry C, et al. Determinants and outcomes of stroke following percutaneous coronary intervention by indication. Stroke 2016; 47(6):1500-7. doi: 10.1161/ STROKEAHA.116.012700

9. Kwok C, Kontopantelis E, Myint P, Zaman A, Berry C, Keavney B, et al. Stroke following percutaneous coronary intervention: type-specific incidence, outcomes and determinants seen by the British Cardiovascular Intervention Society 2007-12. Eur Heart J. 2015; 36(25):1618-28. doi: 10.1093/eurheartj/ehv113

10. Werner N, Bauer T, Hochadel M, Zahn R, Weidinger F, Marco J, et al. Incidence and clinical impact of stroke complicating percutaneous coronary intervention: results of the Euro heart survey percutaneous coronary interventions registry. Circ Cardiovasc Interv. 2013; 6(4):362-9. doi: 10.1161/CIRCINTERVENTIONS. 112.000170

11. Shivaraju A, Yu C, Kattan M, Xie H, Shroff A, Vidovich $\mathbf{M}$. Temporal trends in percutaneous coronary intervention-associated acute cerebrovascular accident (from the 1998 to 2008 Nationwide Inpatient
Sample Database). Am J Cardiol. 2014; 114(2):206-13. doi: 10.1016/j.amjcard.2014.04.044

12. Wong S, Minutello R, Hong M. Neurological complications following percutaneous coronary interventions (a report from the 2000-2001 New York State Angioplasty Registry). Am J Cardiol 2005; 96(9):1248-50. doi: 10.1016/j.amjcard.2005.06.065

13. Varmdal T, Janszky I, Bakken I, Ellekjær H, Fjærtoft H, Håberg S, et al. Percutaneous Coronary Intervention as a Trigger for Stroke. Am J Cardiol. 2017;119(1):35-39. doi: 10.1016/j.amjcard.2016.09.012

14. Kawamura A, Lombardi D, Tilem M, Gossman D, Piemonte T, Nesto R. Stroke complicating percutaneous coronary intervention in patients with acute myocardial infarction. Circ J. 2007; 71(9):1370-5. doi: 10.1253/circj.71.1370

15. Segal A, Abernethy W, Palacios I, BeLue R, Rordorf G. Stroke as a complication of cardiac catheterization: risk factors and clinical features. Neurology 2001; 56(7):975-7. doi: 10.1212/wnl.56.7.975

16. Keeley E, Grines C. Scraping of aortic debris by coronary guiding catheters: a prospective evaluation of 1,000 cases. J Am Coll Cardiol. 1998; 32(7):1861-5. doi: 10.1016/s0735-1097(98)00497-5

17. Jolly S, Cairns J, Yusuf S, Meeks B, Pogue J, Rokoss M, et al. Randomized trial of primary PCI with or without routine manual thrombectomy. N Engl J Med. 2015; 372(15):1389-98. doi: 10.1056/NEJMoa1415098

18. Büsing K, Schulte-Sasse C, Flüchter $\mathbf{S}$, Süselbeck T, Haase K, Neff W, et al. Cerebral infarction: incidence and risk factors after diagnostic and interventional cardiac catheterization: prospective evaluation at diffusion-weighted MR imaging. Radiology 2005; 235(1):177-83. doi: 10.1148/radiol.2351040117

19. Didier R, Gaglia M, Koifman E, Kiramijyan S, Negi S, Omar A, et al. Cerebrovascular accidents after percutaneous coronary interventions from 2002 to 2014: incidence, outcomes, and associated variables. Am Heart J. 2016; 172:80-7. doi: 10.1016/j.ahj.2015. 10.019

20. Easton J, Saver J, Albers G, Alberts M, Chaturvedi S, Feldmann E, et al. Definition and evaluation of transient ischemic attack: a scientific statement for healthcare professionals from the American Heart Association/American Stroke Association Stroke Council; Council on Cardiovascular Surgery and Anesthesia; Council on Cardiovascular Radiology and Intervention; Council on Cardiovascular Nursing; and the Interdisciplinary Council on Peripheral Vascular Disease. The American Academy of Neurology affirms the value of this statement as an educational tool for neurologists. Stroke 2009; 40(6):2276-93. doi: 10.1161/ STROKEAHA.108.192218

21. Thygesen K, Alpert J, Jaffe A, Chaitman B, Bax J, Morrow D, et al. Fourth universal definition of myocardial infarction (2018). Circulation 
2018; 138(20):e618-e651. doi: 10.1161/CIR.000000 0000000617

22. Hoffman S, Holmes D, Rabinstein A, Rihal C, Gersh B, Lennon R, et al. Trends, predictors, and outcomes of cerebrovascular events related to percutaneous coronary intervention: a 16-year single-center experience. JACC Cardiovasc Interv 2011; 4(4):415-22. doi: 10.1016/j.jcin.2010.11.010

23. Trujillo P, Batista I, Vignolo G, Durán A, Lluberas R, Vaño E. Protección radiológica al primer operador en procedimientos coronarios por acceso radial derecho. Rev Urug Cardiol. 2015; 30(2):131-8.

24. Kwok C, Kontopantelis E, Myint P, Zaman A, Berry C, Keavney B, et al. Stroke following percutaneous coronary intervention: type-specific incidence, outcomes and determinants seen by the British Cardiovascular Intervention Society 2007-12. Eur Heart J 2015; 36(25):1618-28. doi: 10.1093/eurheartj/ehv113

25. Sirker A, Kwok C, Kotronias R, Bagur R, Bertrand $\mathrm{O}$, Butler R, et al. Influence of access site choice for cardiac catheterization on risk of adverse neurological events: A systematic review and meta-analysis Am Heart J. 2016; 181:107-19. doi: 10.1016/j.ahj. 2016. 06.027
26. Hamon M, Baron J, Viader F, Hamon M. Periprocedural stroke and cardiac catheterization. Circulation 2008; 118(6):678-83. doi: 10.1161/CIRCULATIONAHA. 108.784504

27. Khatri P, Taylor R, Palumbo V, Rajajee V, Katz J, Chalela J, et al. The safety and efficacy of thrombolysis for strokes after cardiac catheterization. J Am Coll Cardiol. 2008; 51(9):906-11. doi: 10.1016/j.jacc. 2007.09.068

28. Camejo C, Legnani C, Gaye A, Arcieri B, Brumett F, Castro L, et al. Unidad de ACV en el Hospital de Clínicas: comportamiento clínico-epidemiológico de los pacientes con ACV (2007-2012). Arch Med Int. 2015; 37(1):30-5.

29. Killip T3rd, Kimball J. Treatment of myocardial infarction in a coronary care unit. A two year experience with 250 patients. Am J Cardiol. 1967; 20(4):457-64. doi: 10.1016/0002-9149(67)90023-9

30. Sociedad Uruguaya de Cardiología. Comité de Hemodinamia. Registro nacional del tratamiento intervencionista del infarto agudo de miocardio en Uruguay (RENATIA). Rev Urug Cardiol. 2006; 21(3):21823. 